\title{
Ontology-Based Support for Hazard and Operability Studies
}

Johannes I. Single*, Jürgen Schmidt, Jens Denecke

CSE-Center of Safety Excellence (CSE-Institut), Pfinztal 76327, Germany

Corresponding Author Email: johannes.single@cse-institut.de

https://doi.org/10.18280/ijsse.100302

Received: 23 December 2019

Accepted: 26 May 2020

\section{Keywords:}

competency questions, HAZOP ontology, ontology-based query answering, ontology design, risk assessment, safety assessment

\begin{abstract}
Ontologies have been used in safety engineering for the representation of knowledge in the form of concepts, facts, and procedures. In this work, an ontology for Hazard and Operability (HAZOP), risk assessment, and related process and plant concepts, is proposed. It is intended to support participants during HAZOP studies. The ontology is designed based on competency questions which can be answered using ontology queries. The developed ontology is applied within a case study to provide knowledge to fill a HAZOP worksheet, including the risk assessment, of a gasoline storage tank system. The results demonstrate that the ontology is well-suited to answer the formulated competency questions correctly. Additionally, metrics have been used to assess the quality of the ontology and to be able to compare ontologies on a quantitative basis. Within future research, the proposed ontology could be enhanced and integrated with an upper ontology.
\end{abstract}

\section{INTRODUCTION}

In the field of safety management and risk assessment, the HAZard and OPerability (HAZOP) technique is a generally accepted hazard evaluation procedure. It is a human-centered moderated brainstorming technique that is used to identify and assess hazards and operability issues that can occur in processes and plants. The procedure is time and laborintensive, and the results depend on the composition of the participants, moderation of the study, personal experience, level of training, communication and safety culture.

Within the field of safety engineering, various research groups have been dealing with the topic of knowledge-based systems and ontologies. Knowledge-based systems are computer systems that utilize a knowledge-representation to provide expert knowledge or draw conclusions.

Within computer science, ontologies can be understood as knowledge models that are used for the representation of knowledge. Gruber described ontologies as an "explicit specification of a conceptualization" [1]. This means a set of concepts and the relations between them are described unambiguously. Domain-specific concepts, facts, and procedures can be put in context with each other. This can contribute to establish a common understanding of information and make knowledge available to humans and machines.

Ontologies have been developed in the safety engineering domain within research approaches, such as:

- training system for occupational risk prevention [2],

- construction safety ontology for automated safety planning for occupational hazard analysis [3],

- ontology for the process safety management (PSM) domain [4],

- ontology for the process safety domain [5],
- a framework to support fault prognosis in gas and oil production plants [6],

- $\quad$ conceptual ontology for HAZOP studies [7],

- ontology for process equipment related incident investigations [8],

- ontology-based framework for process supervision in chemical plants [9],

- decision support system for chemical substances based on ontologies [10],

- ontology-based chemical accident database [11].

Other research groups have used ontologies intending to automate HAZOP studies [12-15].

Most of these approaches have different aims and scopes, while the applicability and reusability of ontologies depend on the underlying principles, level of details, concepts, context, and ontology language.

Even though in the field of process safety, ontologies have been used by different researchers, only partial aspects of HAZOP concepts are covered in the approaches. Thus, no ontology model has been published that can be directly used to support practitioners within HAZOP studies.

\subsection{Objectives of this research}

The focus of this research is the development of an ontology that represents a knowledge model from the HAZOP domain. The idea is to provide a knowledge representation that can be queried to support participants during HAZOP studies and the preparation of HAZOP worksheets. An approach not yet followed by other researchers in the field of HAZOP is the formulation and formalization of competency questions to be answered by the ontology. Therefore a detailed description of HAZOP and risk assessment concepts and the relations between these concepts and other HAZOP-related concepts, such as units, processes, and substances, is provided. On the 
one hand, a suitable ontological model shall be presented, and on the other hand, the application shall be demonstrated. The evaluation of the ontology and the obtained query results are to be evaluated based on the quality of the results obtained and specific ontology metrics.

\section{KNOWLEDGE AND ONTOLOGY DESIGN}

Within the VDI 5610 guideline, the terms "data", "information", and "knowledge" are differentiated [16]. The term, "data", is described as objective facts without context, while "information" is described as structured data with relevance and purpose. The term 'knowledge' is described as linked information that can be used to draw comparisons, establish links, and make decisions [16].

There are various formalisms for the representation of data, information, and knowledge. The formalisms include glossaries, data dictionaries, thesauri, taxonomies, database schemata, data models, and formal ontologies [17].

The most advanced group of knowledge representation formalisms are formal ontologies, which are formal definitions of domain concepts (classes), relationships between the concepts, and additional constraints. Due to their formal semantics, ontologies can be used to represent knowledge unambiguously.

\subsection{Classification and design consideration of ontologies}

Ontologies can be classified by their expressiveness. A lightweight ontology contains essential concepts and relationships, while a heavyweight ontology also contains formal axioms and constraints besides concepts and relationships.

Ontologies can be divided into top-level, domain, task, and application ontologies [18]. Top-level or upper-level ontologies are used to describe very general concepts that are common across various domains [19]. Domain or task ontologies contain generic concepts, e.g., concepts from process and plant safety. Application ontologies are used for specific application use cases, e.g., vocabulary matching between software packages.

After the conceptualization, the knowledge model must be formalized to be machine-processable, using an ontology language. The most common ontology languages are the Resource Description Framework (RDF) schema and the Web Ontology Language (OWL) [20]. The RDF language can be used to describe classification hierarchies and their relationships. There are three variants of OWL with different levels of expressiveness: OWL Lite, OWL-DL, and OWL Full. OWL Lite can be used to describe classification hierarchies and simple constraints. OWL DL is a compromise between expressiveness and efficient reasoning. Description logics (DL) are knowledge representation languages that are equipped with formal semantics [20]. OWL Full supports maximum expressiveness with no computational guarantees [20]. OWL is the standard modeling language for ontologies as recommended by the World Wide Web Consortium (W3C) in 2004 [21]. The essential elements of the OWL language are classes (concepts), properties (relationships), individuals (instances), and restrictions (axioms) that are used to increase the semantic unambiguity. Classes are representations of concepts and sets that contain individuals. Individuals are instances of classes. Object properties are used to relate individuals to each other, and define inheritance relations (is_a) to connect classes and subclasses.

After discussing primary considerations, formalization and formal languages, the ontology development process can be divided into several phases [22]:

- Specification: define the purpose, level of formality and scope,

- Conceptualization: structure the domain knowledge, build a conceptual model,

- Formalization: formalize the conceptual model, e.g., using description logic,

- Integration: reuse of concepts from other ontologies,

- Implementation: using a formal ontology language,

- Evaluation: check definitions and integrate with a suitable upper ontology,

- Documentation: make it reusable and sharable.

\section{ONTOLOGY FOR HAZOP STUDIES}

The purpose of the ontology must be clear, including the use cases, users, and applications. With the help of competency questions, the scope of the ontology can be clearly defined. This means a set of competency questions must be answered using the ontology. The conceptualization process includes the identification of relevant concepts, their relations, and granularity. It is crucial to model relations between concepts semantically correct and in a consistent way in order to be able to answer the competency questions. The intended meaning must be described unambiguously. In this work, the Web Ontology Language (OWL-DL) is used. The ontology modeling, debugging, and visualization is done using the Protégé ontology editor [23].

\subsection{Purpose and components of the developed ontology}

The developed ontology for HAZOP studies (OntoHazop) represents a knowledge model to support HAZOP participants in conducting HAZOP studies. With the help of the ontology, HAZOP specific competency questions are to be answered in order to support the creation of HAZOP worksheets. The developed ontology can be classified as an ontology with the generic concepts of a domain ontology.

Within the knowledge model, concepts from the field of process and plant safety, including HAZOP, risk assessment, and process and plant-specific concepts are considered. The main concepts are defined in Table 1. A conceptual model of the developed ontology is shown in Figure 1. It includes the main concepts and relations between them. Within the OWL language relations between concepts are called object properties. For example, in Figure 1, the concept "Deviation" is related to "Parameter" with the object property "has_parameter" and to "Guideword" with the object property "has_guideword".

\subsection{HAZOP concepts}

The HAZOP technique is a scenario-based hazard identification procedure. A scenario can be composed of the typical HAZOP concepts, such as deviation, cause, consequence, safeguard (see Table 1). These concepts correspond to the typical HAZOP worksheet categories. One of the first steps within HAZOP studies is the division of the 
process plant into study nodes. These consist of sections of process units and equipment.

Deviations are used to describe process deviations or other deviations from the design intention. Deviations are applied to the study nodes and are composed of guidewords and parameters. For example, within the ontology, this would mean there are object properties between the deviation "HighPressure", the guideword "More" and the parameter "Pressure".

Causes represent reasons for the deviations and consequences possible results. As shown in Figure 1, causes and consequences are connected to the unit concept. Also, the substance and consequence concepts are connected. For instance, the substance property has a direct influence on the consequences.

Safeguards are used to either prevent potential causes or mitigate potential consequences. Accordingly, in Figure 1, the safeguard concept is connected with the cause and consequence concept.

A visualization of the concepts of the implemented ontology is shown in Figure 2. It was visualized using Protégé and OntoGraf [23].

Table 1. Description of the main concepts

\begin{tabular}{|c|c|c|}
\hline Category & Concept & Description \\
\hline \multirow{6}{*}{ HAZOP } & Parameter & Describes parameters/system properties. \\
\hline & Guideword & Concepts to describe deviation perspectives systematically. \\
\hline & Deviation & $\begin{array}{l}\text { Describes deviation from the design intent within nodes. It is composed of the } \\
\text { guidewords and (process) parameters, }\end{array}$ \\
\hline & Cause & Describes causes that lead to deviations in the intention within the node. \\
\hline & Consequence & Describes consequences that arise from the deviations. \\
\hline & Safeguard & Describes safeguards against scenarios. \\
\hline \multirow{3}{*}{ Risk assessment } & Likelihood & Describes the likelihood of a cause qualitatively. \\
\hline & Severity & Describes the severity of a consequence qualitatively. \\
\hline & Risk & Resulting from a likelihood and a severity. \\
\hline \multirow[b]{2}{*}{ Process and plant } & Unit & Describes involved units, such as equipment, piping, instrumentation. \\
\hline & $\begin{array}{l}\text { Process } \\
\text { Substance }\end{array}$ & $\begin{array}{c}\text { Describes safety-relevant concepts of processes that take place in the equipment. } \\
\text { Describes involved substances and properties. }\end{array}$ \\
\hline
\end{tabular}

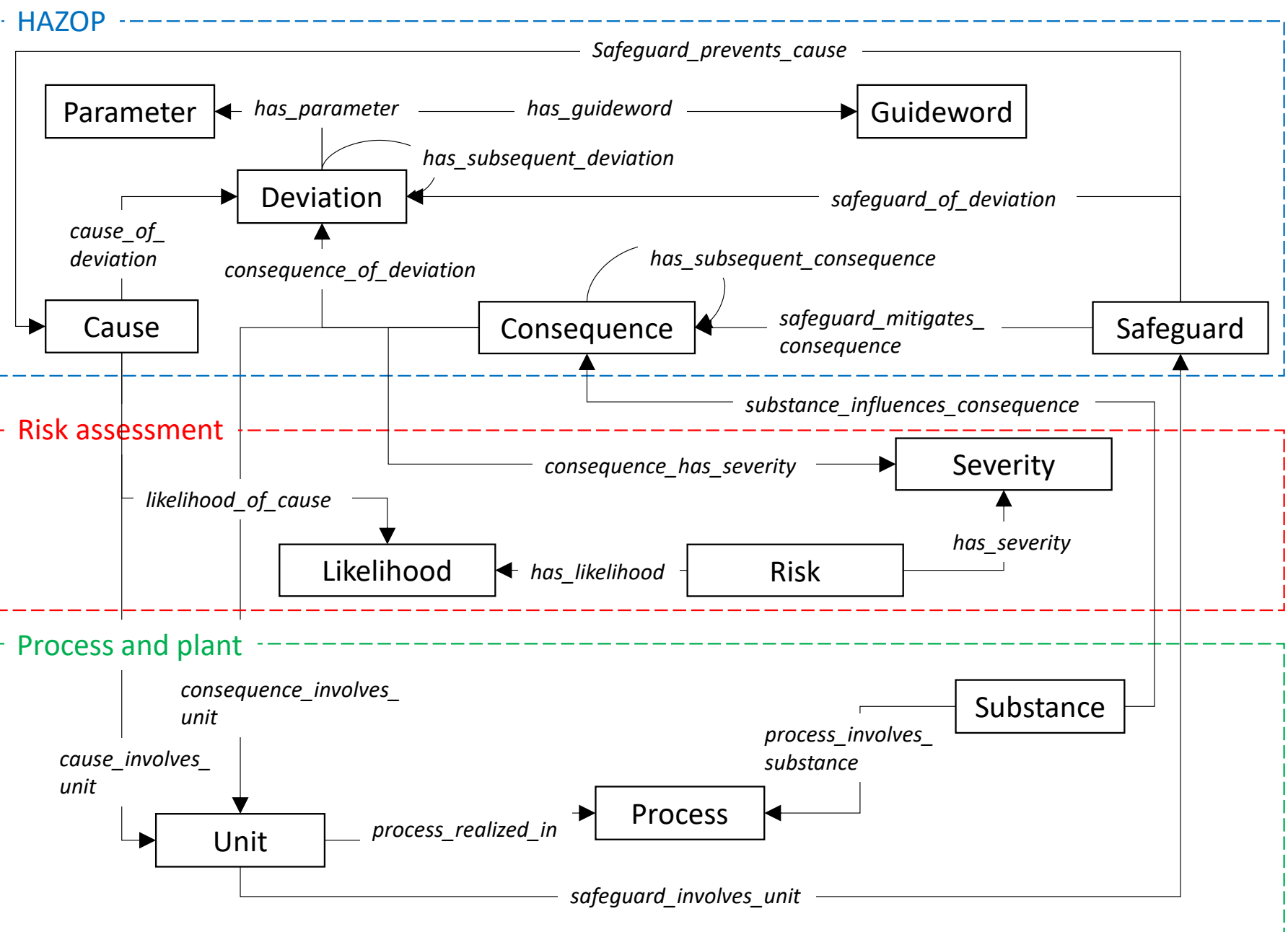

Figure 1. Main concepts and relations of the proposed ontology 
Table 2. Qualitative risk matrix

\begin{tabular}{cc|ccccc}
\hline & \multicolumn{5}{c}{ Severity } \\
\cline { 3 - 6 } & very likely & negligible & minor & major & critical & catastrophic \\
& likely & low & moderate & moderate & high & very high \\
Likelihood & possible & low & moderate & moderate & moderate & high \\
& unlikely & very low & low & moderate & moderate & moderate \\
& Very Unlikely & very low & very low & low & low & moderate \\
\hline
\end{tabular}

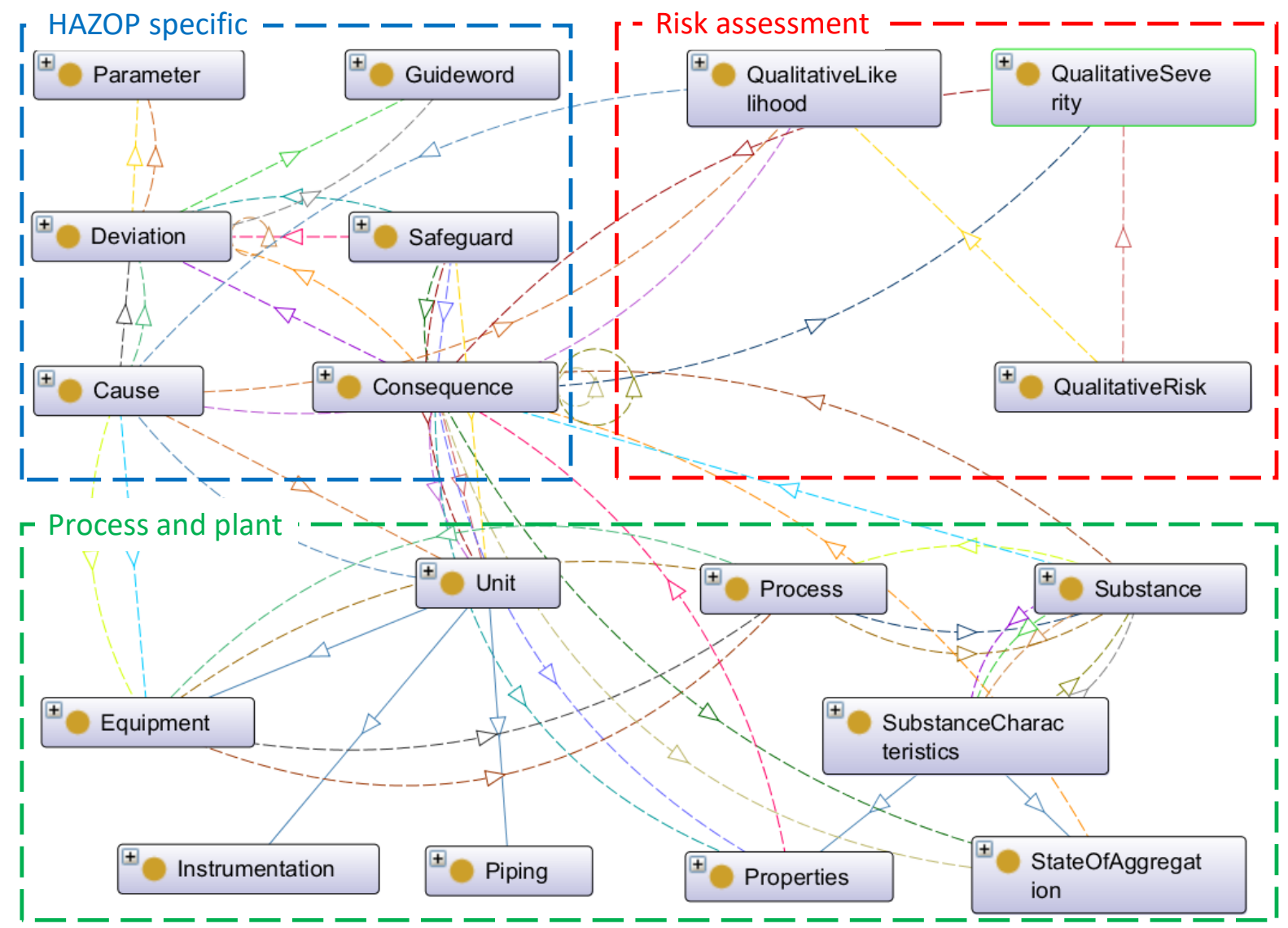

Figure 2. Main concepts visualization using Protégé and OntoGraf

\subsection{Risk assessment concepts}

Behind the risk concepts, there is a qualitative risk matrix that is shown in Table 2. It is composed of five risk ranking categories, such as "very high", "high", "moderate", "low", "very low", five categories of likelihood, and five levels of severity.

Within the exemplary matrix, multiple combinations of likelihood and severity lead to the same risk. The risk ranking categories usually depend on the industry in which risks are assessed and internal company guidelines.

The risk ranking categories can be used to qualify the (raw) risk of a scenario. Therefore, a likelihood is assigned to a cause, while severity is assigned to a consequence. Within the ontology, this is taken into account by assigning a likelihood to each cause (compare Q6 in Table 3). The equipment (or unit) involved must also be considered. Similarly, the consequences have been assigned a severity (compare Q7 in Table 3).

Thus the risk category for a scenario can be determind based on the likelihood and severity. Within the scope of this work, the risk assessment is qualitative (see Figure 2), but it can also be quantitative. Once the risk for the scenario has been determined, safeguards must be established to reduce the risk.
Risk ranking categories, such as "very low" require no risk mitigation and are acceptable as is.

This risk matrix was modeled within the ontology. An exemplary formal DL-based definition of the risk class "High" can be represented as follows:

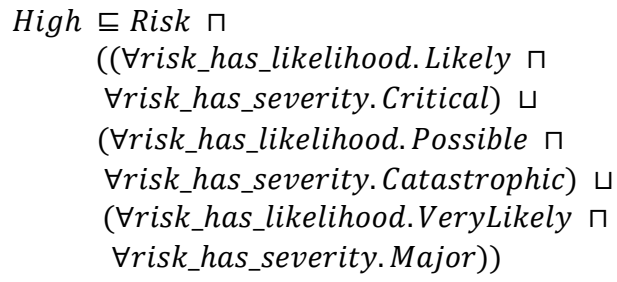

\subsection{Process and plant concepts}

Beside HAZOP and risk assessment concepts, process and plant concepts are considered. Unit-specific concepts are needed to identify plausible causes or appropriate safeguards. Also, the likelihood of causes depends on the process unit and equipment. The unit-specific concepts include piping (e.g., pipes, valves), instrumentation (e.g., sensors, process control), and equipment (e.g., vessel, pumps). 
For the identification of potential consequences, information on the process and the substances involved is required. The consequence directly influence the assessment of the severity. Hence, the characteristics of involved substances, such as (hazardous) properties or state of aggregation, are considered. For instance, the hazardous attributes of a substance could lead to more specific consequences.

The links in Figure 2 show the dependencies between concepts. For instance, the concept "Consequence" is strongly linked to the concepts "Unit" and "Substance".

The process and plant concepts are used to represent the study nodes of the HAZOP study.

\subsection{Querying the ontology}

After the design and formalization of the ontology, the knowledge it contains must be made usable using reasoners. Reasoners are software packages that are used for classification, ensuring logical consistency, and query answering. They infer logical consequences from the asserted facts and axioms of the ontology. Therefore, the reasoning is based on the inference rules that are specified by the ontology language. The HermiT reasoner is integrated with Protégé and was used in this work [24].

In Figure 3, exemplary queries and query answers are shown that are created using the Protégé editor. The drawn conclusions are made available in the form of query answers. All three queries have different scopes, such as the inference of a HAZOP deviation, subsequent consequence, and the likelihood of a cause.

In order to use ontologies within HAZOP studies, HAZOP relevant competency questions must be answered with the help of ontologies. Accordingly, competency questions are needed to help create HAZOP worksheets. In Table 3, eight different competency questions are formulated for that purpose.

With the help of the competency questions (see Table 3), causes (Q1), consequences (Q2, Q3), and safeguards (Q4, Q5) can be identified. Also, the likelihood of specific causes under consideration of the associated equipment can be queried (Q6). Additionally, the severity of the consequence can be queried (Q7). Based on the likelihood and the severity, the (qualitative) risk can be determined $(\mathrm{Q} 8)$.

After the formulation of the relevant competency questions, they need to be formalized into queries, compare Table 3.

Besides the formulation of the competency questions, exemplary input for the queries, and the query language syntax of the Protégé ontology editor, which is based on the Manchester OWL syntax is shown in Table 3. Queries can be conducted manually or automatically by computer systems.

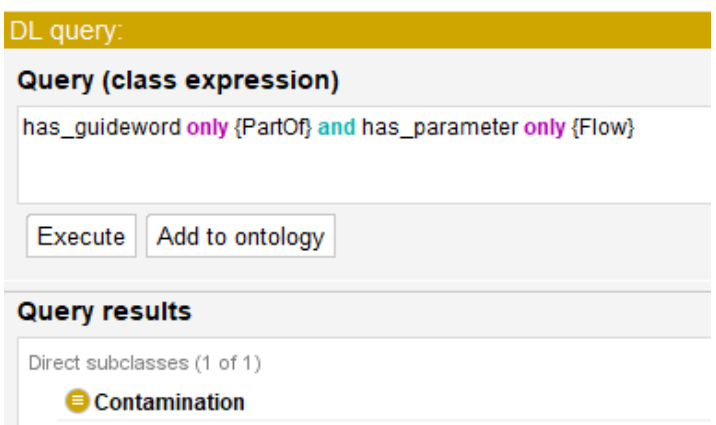

(a) HAZOP deviation

DL_query:
Query (class expression)
is_subsequent_consequence_of only LossOfContainment and
consequence_involves_properties only \{Flammable\}
Execute Add to ontology
Query results
Subclasses (1 of 2)
FlammableSubstanceRelease

(b) Subsequent consequence

DL query:
Query (class expression)
is_likelihood_of_cause only FaultyLevelControl and
cause_involves_equipment only StorageTank
Execute Add to ontology
Query results
Direct subclasses (1 of 1 )
Likely

(c) Likelihood of a cause

Figure 3. Exemplary queries and the corresponding query answers

\section{CASE STUDY: GASOLINE STORAGE TANK}

The developed OntoHAZOP ontology is evaluated through queries to answer competency questions and fill a HAZOP worksheet. A case study was carried out as part of this work that involves a simplified gasoline storage tank system that is shown in Figure 4.

The node under consideration consists of the storage tank, including a pump in the inlet line and several valves. The intention of the node is the storage of liquid gasoline without exceeding a specified pressure.

Plant specific details are described using specific equipment instrumentation, or piping.

In the ontology, the storage tank is modeled as an instance of the "Unit" concepts, including the equipment "FeedPump" and the piping "InletValve", "OutletValve" and "DrainValve". The intention of the storage tank is considered using the "NominalFunction" and "Storage" concept, which is assigned to the "StorageTank" object. The state of aggregation "Liquid" and hazardous attributes "Flammable", "HealthHazard" and "EnvironmentalHazard" of the gasoline are considered.

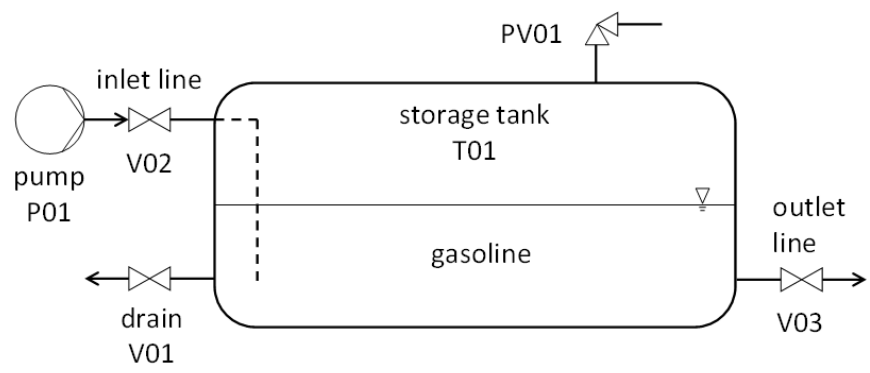

Figure 4. Simplified gasoline storage tank system 
Table 3. Competency questions and query formulation

\begin{tabular}{|c|c|c|c|}
\hline No & Competency question & Input & $\begin{array}{l}\text { Query (syntax) } \\
\end{array}$ \\
\hline Q1 & $\begin{array}{l}\text { What are the potential causes of a } \\
\text { specific deviation in combination } \\
\text { with equipment/piping? }\end{array}$ & $\begin{array}{l}\text { EQUIPMENT: StorageTank } \\
\text { DEVIATION: HighPressure, } \\
\text { HighLevel, LowLevel, } \\
\text { ElsewhereFlow }\end{array}$ & $\begin{array}{c}\text { cause_involves_equipment only EQUIPMENT } \\
\text { and } \\
\text { cause_of_deviation only } \\
\text { DEVIATION }\end{array}$ \\
\hline Q2 & $\begin{array}{l}\text { What are the potential } \\
\text { consequences of a specific } \\
\text { deviation in combination with } \\
\text { equipment/piping? }\end{array}$ & $\begin{array}{l}\text { EQUIPMENT: StorageTank } \\
\text { DEVIATION: NoFlow, LowFlow, } \\
\text { HighPressure, HighLevel, } \\
\text { LowLevel }\end{array}$ & $\begin{array}{c}\text { consequence_involves_equipment only } \\
\text { EQUIPMENT } \\
\text { and } \\
\text { consequence_of_deviation only DEVIATION }\end{array}$ \\
\hline Q3 & $\begin{array}{l}\text { What are the potential subsequent } \\
\text { consequences? }\end{array}$ & CONSEQUENCE: Overfilling & $\begin{array}{c}\text { has_subsequent_consequence some } \\
\text { CONSEQUENCE }\end{array}$ \\
\hline Q4 & $\begin{array}{c}\text { What are safeguards of a specific } \\
\text { deviation in combination with an } \\
\text { equipment/piping? }\end{array}$ & $\begin{array}{c}\text { EQUIPMENT: StorageTank } \\
\text { DEVIATION: HighPressure, } \\
\text { HighLevel, ElsewhereFlow, ... }\end{array}$ & $\begin{array}{l}\text { safeguard_involves_equipment only } \\
\text { EQUIPMENT } \\
\text { and safeguard_of_deviation only DEVIATION }\end{array}$ \\
\hline Q5 & $\begin{array}{l}\text { What are safeguards that mitigate a } \\
\text { particular consequence in } \\
\text { combination with an } \\
\text { equipment/piping? }\end{array}$ & $\begin{array}{l}\text { CONSEQUENCE: } \\
\text { Overfilling, FireHazard, Release, } . . \\
\text { EQUIPMENT: StorageTank }\end{array}$ & $\begin{array}{l}\text { safeguard_mitigates_consequence only } \\
\text { CONSEQUENCE and safeguard_involves } \\
\text { equipment only EQUIPMENT }\end{array}$ \\
\hline Q6 & $\begin{array}{l}\text { What is the likelihood of a cause in } \\
\text { combination with } \\
\text { equipment/piping? }\end{array}$ & $\begin{array}{c}\text { CAUSE: } \\
\text { LeakingDrainValve, } \\
\text { FaultyLevelControl, etc. } \\
\text { EQUIPMENT: } \\
\text { StorageTank }\end{array}$ & $\begin{array}{l}\text { is_likelihood_of_cause only CAUSE and } \\
\text { cause_involves_equipment only EQUIPMENT }\end{array}$ \\
\hline Q7 & $\begin{array}{l}\text { What is the severity of a particular } \\
\text { consequence? }\end{array}$ & $\begin{array}{l}\text { CONSEQUENCE: } \\
\text { Release, FireHazard, ... } \\
\text { LIKELIHOOD: }\end{array}$ & $\begin{array}{l}\text { is_severity_of_consequence only } \\
\text { CONSEQUENCE }\end{array}$ \\
\hline Q8 & $\begin{array}{l}\text { What is the risk based on the } \\
\text { likelihood and the severity? }\end{array}$ & $\begin{array}{l}\text { Likely, Possible, Unlikely, ... } \\
\text { SEVERITY: } \\
\text { Minor, Major, Negligible, } \ldots\end{array}$ & $\begin{array}{l}\text { has_likelihood only LIKELIHOOD and } \\
\text { has_severity only SEVERITY }\end{array}$ \\
\hline
\end{tabular}

Table 4. Query answers regarding the storage tank in the form of a HAZOP worksheet

\begin{tabular}{|c|c|c|c|c|c|c|}
\hline Deviation & Cause (Q1) & $\begin{array}{l}\text { Likelihood } \\
\text { (Q6) }\end{array}$ & Consequence & $\begin{array}{l}\text { Severity } \\
\text { (Q7) }\end{array}$ & Risk (Q8) & Safeguard (Q5) \\
\hline HighPressure & $\begin{array}{l}\text { StrongExternalHeatInput; } \\
\text { ThermalExpansion }\end{array}$ & Unlikely & $\begin{array}{c}\text { Rupture (Q2); } \\
\text { Release (Q3); } \\
\text { FireHazard (Q3) }\end{array}$ & Critical & Moderate & EmergencyVent \\
\hline \multirow[b]{2}{*}{ HighLevel } & $\begin{array}{l}\text { IncreasedInflow; } \\
\text { DecreasedOutflow }\end{array}$ & Possible & Overfilling (Q2) & Major & Moderate & $\begin{array}{l}\text { CollectingBasin; } \\
\text { HighLevelAlarm; }\end{array}$ \\
\hline & FaultyLevelControl & Likely & $\begin{array}{l}\text { Overfilling (Q2); } \\
\text { Release (Q3); } \\
\text { FireHazard (Q3) }\end{array}$ & Critical & High & $\begin{array}{l}\text { GasDetectionSystem; } \\
\text { RegularControlVisits; } \\
\text { FireAlarmSystem }\end{array}$ \\
\hline Contamination & $\begin{array}{c}\text { Foreign- } \\
\text { SubstanceEntering }\end{array}$ & Possible & $\begin{array}{c}\text { Decreased- } \\
\text { ProductQuality (Q2), }\end{array}$ & Major & Moderate & RegularTankCleaning \\
\hline \multirow{2}{*}{ LowLevel } & $\begin{array}{c}\text { OutletValveWrongly- } \\
\text { Opened } \\
\text { ReducedInflow; } \\
\text { IncreasedOutflow }\end{array}$ & Possible & DeliveryFailure (Q2) & Negligible & Low & LowLevelAlarm \\
\hline & MajorLeakage, & Unlikely & $\begin{array}{l}\text { LossOfContainment } \\
\text { (Q2); } \\
\text { Release (Q3); } \\
\text { FireHazard (Q3) }\end{array}$ & Critical & Moderate & $\begin{array}{l}\text { GasDetectionSystem; } \\
\text { Regular-ControlVisits; } \\
\text { FireAlarmSystem }\end{array}$ \\
\hline ElsewhereFlow & $\begin{array}{l}\text { OpenDrainValve } \\
\text { LeakingDrainValve } \\
\text { MinorLeakage }\end{array}$ & $\begin{array}{l}\text { Possible } \\
\text { Unlikely } \\
\text { Possible }\end{array}$ & $\begin{array}{l}\text { LossOfContainment } \\
\text { (Q2); } \\
\text { Release (Q3); } \\
\text { FireHazard (Q3) } \\
\end{array}$ & Critical & $\begin{array}{l}\text { Moderate } \\
\text { Moderate } \\
\text { Moderate }\end{array}$ & $\begin{array}{c}\text { GasDetection-System; } \\
\text { RegularControlVisits; } \\
\text { FireAlarmSystem }\end{array}$ \\
\hline
\end{tabular}

\subsection{Generating HAZOP results}

Ontology queries can be used to extract knowledge from the ontology to fill HAZOP worksheets. The query answers correspond to the answers to the competency questions. The results generated using the ontology are presented in a HAZOP worksheet in Table 4 . The table contains information on which queries from Table 3 led to the results. The table contents correspond directly to the identified classes of the ontology and correspond to the query answers.
The causes are inferred using query Q1 (compare Table 3) with the equipment "StorageTank" and the deviations from the deviation column as an input. The likelihood of causes is inferred using query Q6.

Potential consequences are inferred based on query Q2, taking into consideration the associated equipment "StorageTank" and deviations from the deviation column in Table 4. After some potential consequences are identified, query Q3 is used to infer potential subsequent consequences. For instance, as a primary consequence, "LossOfContainment" 
is detected, the secondary consequence could be "FireHazard". Thus it is possible to identify causal consequences chains. The severity of a consequence is inferred using query Q7.

When determining the likelihood (see Table 3, Q6), both the cause and the equipment (or piping, or unit) are taken into account, because the likelihood depends strongly on the equipment. The severity of the consequence is not directly dependent on the equipment (or piping or unit) but on the type of consequence, e.g., release of flammable liquid. Based on the likelihood and the severity, the risk of a scenario can be identified using query Q8. The risk assessment is based on the qualitative risk matrix from Table 2 .

The safeguards can be inferred based on the equipment and a deviation using query Q4 and based on consequence and the equipment using query Q5. The Safeguards are proposals and still require expert judgment. Based on the proposed ontology, further competency questions can be formulated to identify preventive safeguards based on the cause and the process unit.

The results in Table 4 show that HAZOP results based on ontologies can be generated using the formulated competency questions and formalized queries.

In addition to the qualitative presentation of risk, it is also possible to present risks in quantitative terms. For example, the scenario that a gasoline tank leaks and ignition occurs a frequency of $10^{-6}$ per year can be assumed [25]. The ontology language OWL DL is also suitable for representing numerical values in the form of data properties.

\section{EVALUATION OF THE ONTOLOGY STRUCTURE}

Competency questions can be used as a guide to assess the quality of the query answers. If the way the questions are answered, turn out as desired, the structure is principally suitable to deliver these answers. In case the completeness is inadequate, the ontology must be extended without changing the main concepts or relationships. In case the answers are too shallow or ambiguous, the structure must be changed fundamentally. Additionally, the opinion on the sufficiency of the answers to the competency questions may vary between domain experts.

Besides, different quantitative metrics can be used to evaluate ontologies. Schema metrics can be used to evaluate the design of the ontology and the inherent capability for knowledge representation. Tartir et al. proposed multiple metrics in their ontology evaluation tool OntoQA [26].

The presented ontology currently consists of 166 classes, 1504 axioms, and 48 object properties, and 183 annotations. The degree of cross-linking of the classes can be seen in Figure 2.

The relationship richness (RR) is calculated from the noninheritance relations $P$ (e.g., object and data properties) and the inheritance relationships $H$ (e.g., parent-child object relationship):

$$
R R=\frac{|P|}{|H|+|P|}=0.196
$$

The RR score of the proposed ontology is 0.196 . Tartir et al. state that an ontology with only inheritance relationships usually conveys less information [26].

Another metric is the inheritance richness (IR) it is calculated from the inheritance relationships $H$ and the number of classes $C$ as follows,

$$
I R=\frac{|H|}{|C|}=2.753
$$

The IR score of the proposed ontology is 2.753. According to Tartir et al., the IR score is an indicator of how well knowledge is grouped [26]. A low IR score would represent an ontology that contains in-depth specific knowledge. On the contrary, a high IR score indicates an ontology containing broad but little detailed knowledge.

The metrics capture the current state of the development and must be updated every time the ontology is expanded. For simple ontologies, the metrics can be calculated by counting classes and relations, which is not practical for more complex ontologies. Within this work, the ontology metrics have been calculated using the web-based tool OntoMetrics [27].

To evaluate the metrics, they must be related to other ontologies. For this purpose, the upper ontology BFO 2.0 and the domain ontology OSHDO-Core v1.0 from the occupational safety and health domain are compared to the proposed ontology $[28,29]$. The aim of the analysis is not to compare the quality or content of the ontologies, but to draw structural conclusions that can be derived from the metrics. The ontologies have the following metrics:

- $\quad$ BFO (upper ontology), RR: 0.346, IR: 0.971

- OSHDO (domain ontology), RR: 0.2, IR: 0.636

- OntoHazop (ontology), RR: 0.196, IR: 2.753

The RR value of OntoHazop ontology is comparable to that of OSHDO ontology. The value of BFO ontology is higher compared to OSHDO and OntoHazop. This circumstance suggests that the diversity of relations of the BFO ontology is higher compared to OSHDO and OntoHazop. As the BFO is an upper ontology, and the other two ontologies are more specific, this conclusion is plausible.

The IR score of the OntoHazop ontology is higher than that of BFO and OSHDA. From this, it can be concluded that OntoHazop covers a broader range of concepts, while BFO and OSHDA cover more details compared to BFO.

Therefore the ontology metric score also depends on the type of ontology, e.g., upper or domain ontology. Furthermore, metrics can also vary significantly by modeling style, e.g., some researchers use classes rather than individuals (instances).

A general assessment of the ontology solely based on the ontology metrics is not feasible because qualitative aspects such as the ability to answer competence questions are also of relevance. At the same time, metrics provide the possibility to compare ontologies on a quantitative basis directly and track changes in the development.

\section{CONCLUSIONS}

The scope of the ontology for HAZOP studies (OntoHazop) is carefully defined with the help of competency questions to be answered using the ontology. Elsewise, domain specifics could be covered too detailed while others are too shallow. 
Also, necessary relations between concepts could be ignored, which could lead to incomplete answers. After the conceptualization process, the ontology is formalized using the OWL-DL ontology language. Due to its expressiveness, OWL DL is well suited to represent the concepts relevant to this work.

Knowledge can be retrieved via manual queries, e.g., using the ontology editor Protégé. Furthermore, knowledge retrieval can be done by software-based queries that are formulated as a sequence and that are automatically evaluated.

The developed ontology is applied within a case study involving a gasoline storage tank that represents the study node. In this approach, the process unit can be composed of specific equipment, instrumentation, or piping.

The HAZOP worksheet related results demonstrate, that the formulated and formalized competency questions are wellsuited to extract relevant knowledge. It is also considered to formulate further competence questions can be formulated based on the same ontology model in order to obtain more further results. Also, the ontology is suitable for representing concepts for risk assessment, such as the likelihood of causes and severity of consequences. It is also possible to model quantitative concepts using data properties.

Besides the evaluation of the results, ontology metrics have been used to compare different ontologies and to quantify and assess the structure of the designed ontology.

An application in industrial practice requires the integration of ontology and an intelligent query system within a userfriendly application. Such an application would enable engineers in training or non-experts to participate in HAZOP studies, while the results are used as a basis for discussion.

The scope of the ontology could be extended to be able to analyze several units together (upstream and downstream). Also, the substance concepts could be enhanced with information on chemical reactions. In the future, the ontology will be extended by further topics from the HAZOP and risk assessment process and plant safety domain.

\section{REFERENCES}

[1] Gruber, T.R. (1993). A translation approach to portable ontology specifications. Knowledge Acquisition, 5(2): 199-220. https://doi.org/10.1006/knac.1993.1008

[2] Galatescu, A., Alexandru, A., Zaharia, C., Kovacs, S. (2010). Ontology-based modeling and inference for occupational risk prevention. The Fourth International Conference on Advances in Semantic Processing, pp. 205-212.

[3] Zhang, S., Boukamp, F., Teizer, J. (2015). Ontologybased semantic modeling of construction safety knowledge: Towards automated safety planning for job hazard analysis (JHA). Automation in Construction, 52: 29-41. https://doi.org/10.1016/j.autcon.2015.02.005

[4] Tan, X.C., Yew, K.H., Low, T.J. (2012). Ontology design for process safety management. International Conference on Computer and Information Sciences, Kuala Lumpeu (ICCIS), pp. 114-119. http://doi.org/10.1109/ICCISci.2012.6297223

[5] Rodríguez, M., Laguía, J. (2019). An ontology for process safety. Chemical Engineering Transactions, 77: 67-72. https://doi.org/10.3303/CET1977012

[6] Ebrahimipour, V., Yacout, S. (2013). An integrated ontology framework to support fault prognosis system considering environmentally conscious production. 22nd International Conference on Production Research.

[7] Batres, R. (2004). An Ontology Approach to Support HAZOP Studies. Proceedings of the Asian Pacific Confederation of Chemical Engineering congress. https://doi.org/10.11491/apcche.2004.0.466.0

[8] Mohammadfam, I., Kalatpour, O., Golmohammadi, R., Khotanlou, H. (2013). Developing a process equipment failure knowledge base using ontology approach for process equipment related incident investigations. Journal of Loss Prevention in the Process Industries, 26: 1300-1307. https://doi.org/10.1016/j.jlp.2013.07.013

[9] Musulin, E., Roda, F., Basualdo, M. (2013). A knowledge-driven approach for process supervision in chemical plants. Computers and Chemical Engineering, 59:

164-177.

https://doi.org/10.1016/j.compchemeng.2013.06.009

[10] Baumeister, J., Striffler, A. (2015). Knowledge-driven systems for episodic decision support. Knowledge-Based Systems, 88 : http://dx.doi.org/10.1016/j.knosys.2015.08.008

[11] Single, J.I., Schmidt, J., Denecke, J. (2020). Knowledge acquisition from chemical accident databases using an ontology-based method and natural language processing. Safety Science, 129 : 104747. https://doi.org/10.1016/j.ssci.2020.104747

[12] Single, J.I., Schmidt, J., Denecke, J. (2019). State of research on the automation of HAZOP studies. Journal of Loss Prevention in the Process Industries, 62: 103952. https://doi.org/10.1016/j.jlp.2019.103952

[13] Zhao, J., Cui, L., Zhao, L., Qiu, T., Chen, B. (2009). Learning hazop expert system by case-based reasoning and ontology. Computers \& Chemical Engineering, 33: 371-378. https://doi.org/10.1016/j.compchemeng.2008.10.006

[14] Daramola, O., Stålhane, T., Omoronyia, I., Sindre, G. (2013). Using ontologies and machine learning for hazard identification and safety analysis. In: Maalej, W., Thurimella, A.K. (eds.) Managing Requirements Knowledge. Springer: Heidelberg. http://dx.doi.org/10.1007/978-3-642-34419-0_6

[15] Single, J.I., Schmidt, J., Denecke, J. (2019). Computeraided HAZOP studies: Knowledge representation and algorithmic hazard identification. WIT Transactions on the Built Environment, 189: 55-66. https://doi.org/10.2495/SAFE190061

[16] Knowledge management for engineering, fundamentals, concepts, approach. (2009). The Association of German Engineers (VDI). VDI 5610-1.

[17] Uschold, M., Grüninger, M. (2004). Ontologies and semantics for seamless connectivity. ACM SIGMOD Record, 33:

58-64. http://doi.org/10.1145/1041410.1041420

[18] Guarino, N. (1998). Formal Ontology and Information Systems. Proceedings of FOIS'98: 3-15.

[19] Batres, R., West, M., Leal, D., Price, D., Masaki, K., Shimada, Y., Fuchino, T., Naka, Y. (2007). An upper ontology based on ISO 15926. Computers and Chemical Engineering, 31(5-6): 519-534. https://doi.org/10.1016/j.compchemeng.2006.07.004

[20] Antoniou, G., Franconi, E., van Harmelen, F. (2005) Introduction to semantic web ontology languages. In: Eisinger N., Małuszyński J. (eds) Reasoning Web. Lecture Notes in Computer Science, vol 3564. Springer, 
Berlin, Heidelberg. https://doi.org/10.1007/11526988_1

[21] Hitzler, P., Krötzsch, M., Rudolph, S. (2010). Foundations of Semantic Web Technologies, CRC Press, New York, 111-210 http://doi.org/10.1201/9781420090512

[22] Fernández-López, M., Gómez-Pérez, A., Juristo, N. (1997). METHONTOLOGY: From Ontological Art Towards Ontological Engineering. AAAI-97 Spring Symposium Series, Providence, 33-40.

[23] Musen, M.A. (2015). The protégé project: A look back and a look forward. AI Matters, 1(4): 4-12. http://doi.org/10.1145/2757001.2757003

[24] Glimm, B., Horrocks, I., Motik, B., Stoilos, G., Wang, Z. (2014). HermiT: An OWL 2 reasoner. Journal of Automated Reasoning, 53(3): 245-269. https://doi.org/10.1007/s10817-014-9305-1

[25] Uijt de Haag, P.A., Ale, B.J.M. (1999). Guidelines for quantitative risk assessment (Purple Book). Committee for the Prevention of Disasters, the Hague, the Netherlands.

[26] Tartir S., Arpinar, I., Amit, P. (2010) Ontological evaluation and validation. In: Poli, R., Healy, M.,
Kameas, A. (eds.) Theory and Applications of Ontology: Computer Applications. Springer Netherlands, 115-130. http://doi.org/10.1007/978-90-481-8847-5_5

[27] University of Rostock. OntoMetrics - Ontology metrics. https://ontometrics.informatik.uni-

rostock.de/ontologymetrics/, accessed on Dec. 9, 2019.

[28] Basic formal ontology (BFO). https://basic-formalontology.org/, accessed on Dec. 10, 2019.

[29] Ławrynowicz, A., Ławniczak, I. (2015). Towards a core ontology of occupational safety and health. In International Experiences and Directions Workshop on OWL, 134-142. Springer, Cham. https://doi.org/10.1007/978-3-319-33245-1_14

\section{NOMENCLATURE}

C

$\mathrm{H} \quad$ inheritance relations

IR inheritance richness metric

$\mathrm{P}$ non-inheritance relations

RR relationship richness metric 